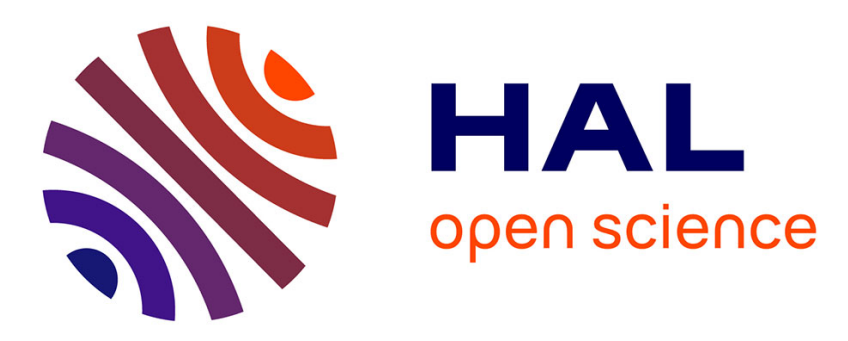

\title{
Analytical Expressions of the Dark Resonance Parameters in a Vacuum Vapor Cell
}

Denis Brazhnikov, Grégoire Coget, Moustafa Abdel Hafiz, Vincent Maurice, Christophe Gorecki, Rodolphe Boudot

\section{- To cite this version:}

Denis Brazhnikov, Grégoire Coget, Moustafa Abdel Hafiz, Vincent Maurice, Christophe Gorecki, et al.. Analytical Expressions of the Dark Resonance Parameters in a Vacuum Vapor Cell. IEEE Transactions on Ultrasonics, Ferroelectrics and Frequency Control, 2018, 65 (6), pp.962 - 972 . hal-02366571

\section{HAL Id: hal-02366571 \\ https://hal.science/hal-02366571}

Submitted on 16 Nov 2019

HAL is a multi-disciplinary open access archive for the deposit and dissemination of scientific research documents, whether they are published or not. The documents may come from teaching and research institutions in France or abroad, or from public or private research centers.
L'archive ouverte pluridisciplinaire HAL, est destinée au dépôt et à la diffusion de documents scientifiques de niveau recherche, publiés ou non, émanant des établissements d'enseignement et de recherche français ou étrangers, des laboratoires publics ou privés. 


\title{
Analytical Expressions of the Dark Resonance Parameters in a Vacuum Vapor Cell
}

\author{
Denis V. Brazhnikov ${ }^{1}$, Grégoire Coget ${ }^{2}$, Moustafa Abdel Hafiz ${ }^{2}$, Vincent Maurice ${ }^{2}$, Christophe Gorecki ${ }^{2}$ and \\ Rodolphe Boudot ${ }^{2}$
}

\begin{abstract}
This article reports a dedicated theoretical and experimental study on the properties (signal amplitude, linewidth) of coherent population trapping (CPT) resonances detected in vacuum vapor cells. Results are presented for conventional singlelambda schemes of atomic energy levels but also for doublelambda schemes, now widely used in various applications including atomic clocks and magnetometers. Approximate compact analytical expressions, valid for a wide range of light wave intensities, i.e. beyond the low intensities or pump-probe regime, have been obtained. Analytical results are found to be in excellent agreement with exact numerical solutions based on the optical Bloch equations. Experimental results, obtained in a Cs vapor microfabricated cell, are reported and found to be in correct agreement with theoretical expressions.
\end{abstract}

\section{INTRODUCTION}

In Coherent Population Trapping (CPT) physics [1], [2], [3], atoms can be prepared in a non-interacting quantum superposition of two hyperfine ground states by coherent interaction with two resonant optical fields coupling a common excited-state in a $\Lambda$-configuration, the main condition being that the two-photon (Raman) frequency detuning must be close to zero [3], [4], [5]. In this so-called dark state, atoms are no longer coupled to light, resulting in enhanced transmission of laser power through the atomic vapor or reduction of the fluorescence emitted by the atoms. The dark resonance line-width is ultimately determined by the lifetime of the hyperfine coherence rather than by the excited state lifetime and can be much smaller than the natural line-width of the optical transition. Consequently, CPT spectroscopy has been widely investigated for precision sensing in view of applications in high-resolution spectroscopy [6], non linear optics, quantum frequency standards [7], magnetometers [8], [9], laser cooling [10], slow light [11] or quantum optics [12]. In most CPT-based atomic devices, light-atom interaction takes place in a vapor cell. In such setups, two main techniques are usually employed to increase the wall-collision induced CPT coherence relaxation time and allow the detection of narrow resonances. The first method consists to dilute the alkali vapor with a pressure of buffer gas [13], that also reduces the Doppler broadening via the Dicke effect [14]. Nevertheless, the presence of buffer gas homogeneously

${ }^{1}$ D. V. Brazhnikov is with Institute of Laser Physics, SB RAS, Novosibirsk 630090, Russia and Novosibirsk State University, Novosibirsk 630090 , Russia. ${ }^{2}$ Other authors are with FEMTO-ST, CNRS, UBFC, ENSMM, 26 Rue de l'Épitaphe, 25030 Besançon cedex, France. D. Brazhnikov was supported by the Russian Science Foundation (no. 17-72-20089). Other authors were supported by LabeX FIRST-TF, Région de Franche-Comté and EquipX OSCIMP. e-mail: brazhnikov@laser.nsc.ru broadens and shifts [15], [16], [17] the optical transition lines, allowing excitation of the dark state towards other excited states and resulting in a decrease of its lifetime. Moreover, the buffer gas induces a temperature-dependent frequency shift of the clock transition that needs to be minimized using a finely-tuned buffer gas mixture [18], [19], [20] or a specific single buffer gas configuration [19], [21]. The second method consists to deposit anti-relaxation coatings such as saturated paraffins [22], [23], octadecyltrichlorosilanes (OTS) coatings [24], [25], [26], or alkene-based materials [27] on the cell inner walls. With such coatings, alkali atoms experience a significant number of atomic-state preserving bounces on the cell walls before destruction of the atom spin orientation (up to $10^{4}$ with paraffins, $10^{6}$ with alkenes and $10^{2}$ to $10^{3}$ with OTS). However, finding a reproducible process to fabricate coated-cells is a challenging task and cell performances developed with an analog process often suffer from a non-negligible dispersion.

The theoretical and experimental study of CPT resonance properties in Doppler broadened media such as evacuated cells has also motivated a significant interest, for fundamental physics but also for potential applications. Line narrowing mechanisms in CPT or electromagnetic induced transparency (EIT) have been reported in Doppler-broadened media. The effect of velocity selective coherent population trapping (VSCPT) or EIT in a Doppler broadened medium has been addressed by Taïchenachev et al. [28], Ye and Zibrov [29], and Javan et al. [30], using only monochromatic laser fields. Failache et al. [31] has investigated theoretically in thin cells (one-dimensional model) the lineshape of CPT resonance with VSCPT in an open atomic system, with Doppler effect on the optical transitions but not on the CPT transition. Some papers have also reported the dependence of the CPT resonance lineshape on the laser linewidth. In [32], it has been reported in a Doppler-free $\Lambda$-system that the CPT resonance is unaffected by the laser linewidth when the driving fields are critically phase correlated. In case of EIT and the use of two independent lasers without phase coherence, Guo et al. [33] showed that the effect of the laser spectral linewidth is equivalent to a relaxation rate between the two ground states. Also, the influence of laser sources with different spectral properties on CPT resonances for atomic clocks applications has been investigated in buffer-gas cells [34]. In 2007, a laser induced line narrowing (LILN) effect of the CPT resonance expected to occur in coated or uncoated alkali vapor cells, especially in small size cells, was reported 
[35]. In this paper, a numerical computation of the CPT resonance was performed in a closed three level atom, in a Doppler broadened vapour in a one-dimensional cell model, taking into account the laser linewidth. From this study, the LILN effect is expected to occur in pure alkali-vapor cells when CPT resonance is excited using a narrow-band laser exhibiting a spectral linewidth narrower than the atom natural linewidth. While no experimental proof of this phenomenon has been reported yet, this effect could be interesting for the development of ultra-compact or even miniature atomic vapor cell clocks [36], [37] based on pure evacuated cells, without buffer gas neither wall-coatings. More recently, the excitation of dark states in evacuated vapor cells has been exploited for the stabilization of laser frequency using original dual-frequency sub-Doppler spectroscopy setups [38], [39].

The CPT phenomenon is in general correctly described by a single $\Lambda$-scheme of atomic energy levels in various regimes of atom-light interaction and resonance observation : stationary (continuous-wave) mode, Ramsey-like technique or active maser approach [2], [4], [5], [40], [41], [7], [42]. The regular $\Lambda$-scheme is presented in Fig. 1a. A significant number of papers has been reported with this approach. Some works have reported analytical expressions for the width and amplitude of the dark resonance in the case of a vacuum vapor cell but for the EIT regime (or pump-probe regime) where one of the laser waves is much weaker than the other one, i.e. $I_{1}<<I_{2}$ where $I_{1}$ and $I_{2}$ stand for the intensity of each light wave respectively [28], [30], [29], [43]. Analytical results have been also reported for the atom at rest [2], [4], [5], [40], [41], [42] or in the case of buffer-gas-filled vapor cells [44], [45], even taking into account the full atomic-level structure [46]. The study of the dark resonance parameters under the CPT regime $\left(I_{1} \sim I_{2}\right)$ in vacuum vapor cells has been investigated in various articles [47], [48].

Despite this well-furnished literature, we have noted that any analytical expressions for the dark resonance parameters (amplitude, linewidth) valid beyond the pump-probe and low saturation limits have been derived and presented yet. Another important point is to note the lack of theoretical expressions describing the parameters of the CPT dark resonance detected in vacuum vapor cells with optimized double- $\Lambda$ CPT pumping schemes (see Fig. 1b), such as lin $\perp$ lin [49], lin \| lin [50], push-pull optical pumping (PPOP) [51], [52] or double-modulation CPT [53]. Such schemes are now widely used with buffer-gas filled cells in concrete applications such as atomic clocks [54], [55] or magnetometers [56].

From this context, the original contribution of this paper is to report a theoretical study on the properties (amplitude, linewidth) of CPT resonances detected in vacuum vapor cells, yielding approximate compact analytical expressions, valid for a wide range of light wave intensities, i.e. beyond the low intensities or pump-probe regime. These clear and compact analytical expressions are found to be in good agreement with exact numerical solutions based on optical Bloch equations. The specific case of a closed double $\Lambda$-scheme, modeling the lin $\perp$ lin pumping configuration when the openness can be neglected, is also considered. Some preliminary experimental tests have been performed in a Cs vapor microfabricated cell in order to evaluate the validity of theoretical calculations.

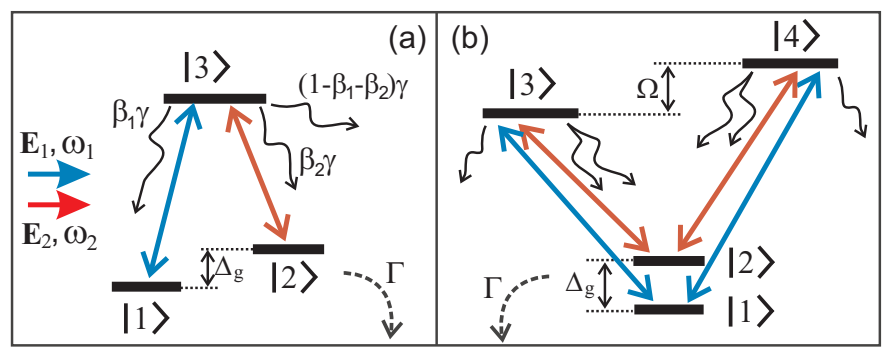

Fig. 1. Single (a) and double (b) $\Lambda$-schemes of atomic energy levels. Wavy arrows denote spontaneous relaxation channels, while $\gamma$ is the total spontaneous relaxation rate from the state $|3\rangle$. In the case of the double $\Lambda$-scheme, each upper-state level relaxes with the rate $\gamma . \Gamma$ stands for the relaxation rate of the ground-state anisotropy, which can be caused by the finite time of flight of an atom through the beam.

\section{THEORETICAL INVESTIGATIONS}

Let us briefly describe the mathematical formalism used in our theory. We consider resonant interaction of atoms with a light field composed of two monochromatic plane waves propagating along the $z$-axis :

$$
E(z, t)=E_{1} e^{-i\left(\omega_{1} t-k_{1} z\right)}+E_{2} e^{-i\left(\omega_{2} t-k_{2} z\right)}+c . c .
$$

The constant phases can be neglected, so that the waves' amplitudes are real numbers. As in [28], we also neglect the residual Doppler broadening of the dark resonance due to the difference of absolute values of the wave vectors, i.e. we take $k_{1} \approx k_{2}=k$. The wave with frequency $\omega_{1}$ induces optical transitions shown as blue arrows in Fig.1, while red arrows correspond to $\omega_{2}$.

In the case of a dilute gas, collisions between atoms can be neglected. Moreover, we do not take into account linear momentum exchange between an atom and a photon, leading to the recoil effect. Thus, the problem can be considered with the help of a one-atom density matrix in the Wigner representation $\widehat{\rho}(z, v, t)$ according to the Lindblad-type equation [57] :

$$
\frac{d \widehat{\rho}}{d t}=-\frac{i}{\hbar}\left[\left(\widehat{H}_{0}+\widehat{V}\right), \widehat{\rho}\right]+\widehat{\mathcal{R}}\{\widehat{\rho}\} .
$$

Here $d / d t=\partial / \partial t+v \partial / \partial z$ with $v$ the projection of atom's velocity on the $z$ axis. The hamiltonian $\widehat{H}_{0}$ describes a free atom, while $\widehat{V}$ is for the atom-field interaction in the dipole approximation. The relaxation operator $\widehat{\mathcal{R}}$ accounts for the spontaneous relaxation with rate $\gamma$ and the corresponding branching ratios $\beta_{1,2}$ (see Fig.1). For the case of an open scheme of levels, we will consider a finite diameter of the light beam. Strictly speaking it means that we should take into account transverse intensity distribution for the waves' amplitudes $E_{1,2}(x, y)$ and include corresponding derivatives to $d / d t$. Instead, the widely used approximation allows to introduce an additional relaxation rate $\Gamma$ responsible for the finite time of 
flight of an atom through the laser beam. Besides, this constant is responsible for the ground-state decoherence rate. Indeed, we consider the case of a regular vacuum vapour cell without any antirelaxation coating of walls. Therefore, there are no ground-state-anisotropy preserving collisions between atoms and the cell walls. This means that we can consider atoms to be distributed isotropically over the ground-state sub-levels when they are out of the light beam. As described further in the text, all three dimensions of the cell are larger than a beam diameter. Moreover, the cell length is large enough not to demonstrate any "thin-cell" physics [31]. To summarize, we can state in our case that atom-wall collisions manifest themselves only via the ground-state thermalization process, which is described by the $\Gamma$ constant.

Before presenting the results of calculations, we note other key assumptions of our theory. Namely, we seek for the steadystate solution of Eq. (2) in the rotating-wave approximation (RWA). Thus, diagonal density matrix elements, responsible for populations of atomic energy levels, can be treated as independent of time. Besides, the power broadening is assumed to be the main process of the dark resonance broadening for the open and closed schemes of energy levels. The time-offlight relaxation process is taken into account in the case of the open $\Lambda$-scheme but is not considered for the closed schemes. We do not use any perturbation theory over the light intensity and the general analytical expressions obtained in the next section are valid for a wide range of wave intensities, as long as the power broadening is smaller than the Doppler width $\Delta_{D}=2 k v_{0}$ (with $v_{0}$ the most probable atom thermal velocity in a gas). The latter condition is regularly satisfied in experiments with CPT in a thermal gas. The last key assumption consists in considering the medium optically thin in order to prevent the solving of a bulky system of Maxwell-Bloch equations [58]. Last words should be said about the light wave frequencies. Usually, due to experimental simplicity reasons, a single laser in combination with a fast electro-optical modulator (EOM) or just a vertical-cavity surface-emitting laser (VCSEL) are used in CPT studies to generate two resonant light waves of frequencies $\omega_{1}$ and $\omega_{2}$. We assume that these frequencies are two \pm 1 sidebands of the frequency spectrum, which are equally distant from the carrier optical frequency $\omega_{0}$. In our calculations, this frequency is taken to be fixed and equal to the averaged frequency of two $\Lambda$-scheme transitions : $\omega_{0}=\left(\omega_{31}+\omega_{32}\right) / 2$. The two-photon (Raman) detuning is defined in $\gamma$ units as $\Delta_{R}=\left(\omega_{1}-\omega_{2}-\Delta_{g}\right) / \gamma$, with $\Delta_{g}$ the frequency difference between the ground-state sublevels (Fig. $1)$. In such real experiments, two frequencies $\omega_{1}$ and $\omega_{2}$ are changed simultaneously but in opposite directions to increase or decrease the two-photon detuning $\Delta_{R}$ and scan the dark resonance.

\section{A. Single-closed $\Lambda$-scheme}

We focus here on a closed scheme of levels, such that the branching ratios (see Fig. 1a) satisfy the condition : $\beta_{1}+\beta_{2}=1$. Also, we assume the branching ratio to be equal to each other. This assumption was also considered in [28], [29], [30] to obtain the expressions of the EIT resonance linewidth.
Assuming this, we consider $\beta_{1,2}=1 / 2$. Note that the equal branching ratio can be easily realized in real experiments with $\mathrm{D}_{1}$ lines of $\mathrm{Rb}$ or $\mathrm{Cs}$ atoms by using the $\sigma^{+} \sigma^{+}$(or $\sigma^{-} \sigma^{-}$) light field configuration. In this paragraph, we also assume that there is no relaxation process for the ground-state anisotropy, i.e. the corresponding relaxation rate $\Gamma=0$. Thus, based on the aforesaid density matrix formalism (see also [2], [4], [5], [41], [42] especially for the CPT studies), the excited state population, i.e. the diagonal density matrix element $\rho_{33}$, is calculated to be expressed as :

$$
W_{\Lambda}\left(x, \Delta_{R}\right) \equiv \rho_{33}=\frac{8 I_{1} I_{2} \Delta_{R}^{2}}{a_{2} x^{2}+a_{1} x+a_{0}}
$$

Here, $x=k v / \gamma$ is the Doppler frequency shift (also in $\gamma$ units) for a moving atom in a gas. The coefficients $a_{j}$ are given by :

$$
\begin{gathered}
a_{0}=4 I_{t}^{3}-4 \Delta_{R}^{2} I_{t}^{2}+\Delta_{R}^{2}\left(1+\Delta_{R}^{2}\right) I_{t}+24 \Delta_{R}^{2} I_{1} I_{2}, \\
a_{1}=4 \Delta_{R}\left(I_{1}-I_{2}\right)\left[\Delta_{R}^{2}-2 I_{t}\right], \\
a_{2}=4 \Delta_{R}^{2} I_{t},
\end{gathered}
$$

where the total intensity $I_{t}=I_{1}+I_{2}$, with saturation parameters $I_{j}=\left(R_{j} / \gamma\right)^{2} \cdot R_{j}=E_{j} d / \hbar$ are the Rabi frequencies, $d$ is the dipole momentum equal to both transitions of the $\Lambda$-scheme. $E_{j}$ are real values of the light wave amplitudes.

Assuming $v=0$ in Eq. (3), we obtain a relatively simple solution for the atom at rest even if the function FWHM $\left(I_{1}, I_{2}\right)$ does not have a compact form. Under the low lightwave intensities limit, we obtain the well-known solution for the linewidth caused by the power broadening mechanism [3], [4], [5] :

$$
\left.F W H M\right|_{I_{1}, I_{2} \ll 1} \sim 4 \gamma\left(I_{1}+I_{2}\right)
$$

This expression is useful to describe the dark resonance in the case where the motion of atoms can be neglected. Besides, the similar linear dependence shown in (7) is also valid for a thermal gas of atoms under the low intensity regime. Also, expression (7) can be obtained from a more general one derived in [42] for atoms at rest. Relatively simple analytical expressions can be obtained in the case of buffergas-filled vapor cells [44], [7], [46]. However, it is important for fundamental laser spectroscopy and many applications of the dark resonances to figure out any explicit analytical expressions in the case of evacuated cells. To achieve this goal, expression (3) is averaged over the Maxwellian velocity distribution :

$$
\left\langle W_{\Lambda}\right\rangle_{v}=\frac{1}{\sqrt{\pi} v_{0}} \int_{-\infty}^{\infty} W_{\Lambda} e^{-v^{2} / v_{0}^{2}} d v
$$

We consider (8) to be the expression of a spectroscopic signal. The transmission of the light beam through an optically thin medium can be easily expressed via $\left\langle W_{\Lambda}\right\rangle_{v}$.

This spectroscopic signal can be obtained by using Eq. (8) and integrating analytically the expression (3). This procedure was applied by other authors and the result is well-known (see for instance [28]). Nevertheless, with this approach, the precise analytical result is expressed via the special error 
function $\operatorname{Erf}(x)$, which cannot be expressed via elementary functions. Moreover, such precise solutions are quite bulky [28]. All these factors make difficult to obtain explicit and compact analytical expressions for the dark resonance parameters (amplitude and linewidth). For this purpose, several approximations are regularly applied. In general, authors try to use an approximate expression for the error function or try to find the most appropriate fitting function for the Gaussian profile in the integral of exp. (8) (for instance, see [30]). Further simplification assumptions are even often considered. A relevant example is to study the problem under the pumpprobe regime, assuming that one of the waves (probe) is much weaker than the other one. This regime is often treated in the case of electromagnetically induced transparency (EIT) where only a probe-wave first-order analysis is valid [28], [29], [30]. In the case where both light field components are weak enough, the optical Bloch equations can be expanded in a series over the small parameter $\xi_{0}$, treated as the "time-offlight" saturation parameter :

$$
\xi_{0}=\gamma \tau I \ll 1
$$

with $\tau=\Gamma^{-1}$ the average time of flight of an atom through the beam. This method has appeared to be powerful enough to obtain the analytical expression for the spectroscopic signal, even in the general case of an optical dipole transition $F_{g} \rightarrow F_{e}$ with degenerate energy levels [59]. However, in this case, the results are valid for quite narrow light beams and weak light fields. Moreover, the signal is also expressed here via the error function, not convenient to obtain compact formula for the resonance parameters. Following all aforesaid, we can state that no compact and clear solutions describing the dark resonance parameters in a thermal vapor cell valid beyond the perturbation theory approach have been obtained yet.

To solve this issue, we do not seek for some approximate formula of the final spectroscopic signal $\left\langle W_{\Lambda}\right\rangle_{v}$. Instead, we try to use the most appropriate approximation for the Gaussian profile standing in Eq. (8). This approach is similar to the one used in [30] for the pump-probe regime and it implies using a Lorentzian profile instead of a Gaussian profile. This approximation (the Lorentzian function exhibits longer "tails" than the Gaussian profile) can help to obtain explicit and compact analytical expressions for the dark resonance parameters. Two options are possible to check the validity of the approximation. The first one consists in providing different results on the same plot, demonstrating the results of approximate analytical expressions together with results of exact numerical calculations with the Gaussian profile fully taken into account. The second way consists in conducting an experiment. In this paper, both options are explored and discussed.

Let us omit routine calculations just for shortness. Only the situation where $I_{1}=I_{2}=I$ is considered. This regime is of relevant practical interest and can be easily realized with the help of $\mathrm{D}_{1}$ lines in $\mathrm{Rb}$ or $\mathrm{Cs}$ atoms irradiated by the $\sigma^{+} \sigma^{+}$(or $\sigma^{-} \sigma^{-}$) light field configuration. The final approximate formula for the dark resonance linewidth in a thermal gas is given to be :

$$
F W H M \approx \frac{8 \gamma}{x_{0} f_{1}} I
$$

with $x_{0}=k v_{0} / \gamma$ the Doppler halfwidth in $\gamma$ units. The dimensionless functions of $I$ have been introduced such as :

$$
f_{1}(I)=\sqrt{\frac{2}{\pi}\left[1+f_{0}(I)-\sqrt{1+2 f_{0}(I)}\right]}
$$

with :

$$
\begin{aligned}
& f_{0}(I)=4 \eta(1+\eta) \\
& \eta=\frac{\sqrt{\pi}}{2 x_{0}} \sqrt{1+12 I}
\end{aligned}
$$

The approximation (10) with functions (11-13) is not so bulky and can be used to estimate the linewidth. By continuously simplifying Eq. (10), we obtain the pretty simple approximation :

$$
F W H M \approx \frac{4 \gamma I}{\sqrt{1+12 I}}
$$

For moderate and relatively high intensities $(12 I \gg 1)$, a simple square-root dependence is obtained :

$$
F W H M \approx 2 \gamma \sqrt{I / 3}
$$

Approximate FWHM expressions described in (10) and (14) are plotted on Fig. 2. Expression (10) is found to be a quite good approximation for FWHM in a wide range of light wave intensities (compare solid black and dashed red curves). The simple formula given in (14) yields also a quite acceptable result (dash-dotted blue curve). On Fig. 2, the linear law from Expr. (7) is reported (almost vertical short-dotted black line). It is well seen that the linewidth starts to deviate from the linear dependence even at low intensities, i.e. $I \ll 1$. In our case, expression (7) works correctly only for $I \lesssim 10^{-3}$. This behavior was discussed in details in [28], [29], [30] for the EIT regime and can be considered as a "Doppler narrowing" effect. The strength of this effect demonstrates one more time how considerable the influence of atomic motion is.

In this paragraph, the decoherence effects in the ground state have been neglected. Thus, the fluorescence signal described in (8) is null under the two-photon resonance condition $\Delta_{R}=$ 0 . Therefore, the amplitude of the dark resonance coincides with the maximum value of the fluorescence signal. At equal Rabi frequencies $R_{1}=R_{2}=R$, this maximum is reached at $\Delta_{R}= \pm 2 R$ and brings the resonance amplitude to be :

$$
A \approx \frac{\pi}{x_{0}^{2} \eta(1+\eta)} I
$$

with $\eta$ described in (13).

In the linear case of very weak intensities $(12 I \ll 1)$, we obtain : 


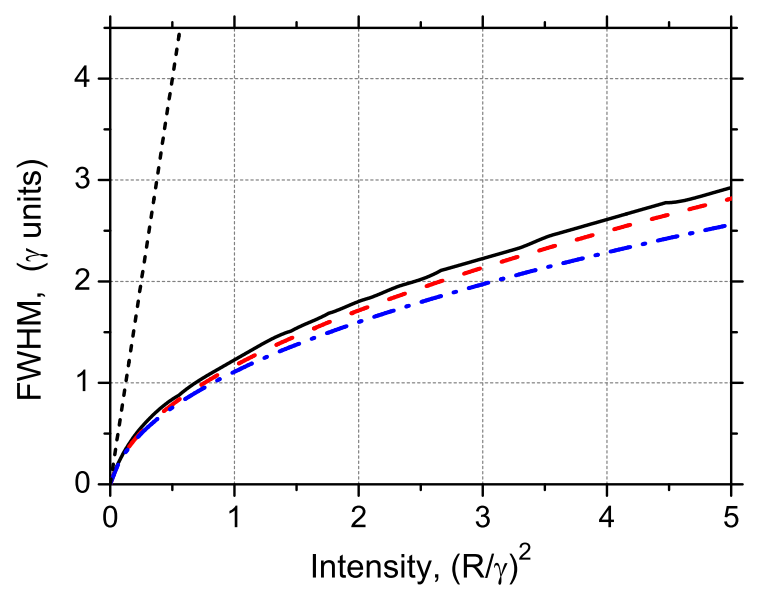

Fig. 2. FWHM of the dark resonance in a vacuum vapor cell for a single closed $\Lambda$-scheme versus the light-wave intensity. The Doppler width $k v_{0}$ equals $50 \gamma$ (typical for the experiments with thermal alkali-metal atoms). The solid line corresponds to the numerical calculation based on accurate expressions (3) and (8). The dash-dotted blue line corresponds to the simplest approximate expression given in (14). The dashed red line stands for the formula (10). The black short-dashed line describes the case of atoms at rest.

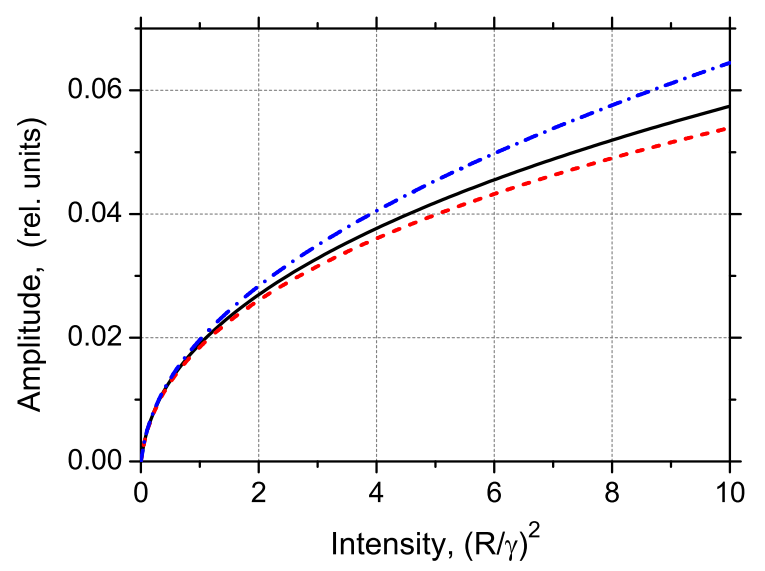

Fig. 3. Amplitude of the dark resonance in a vacuum vapor cell for a single closed $\Lambda$-scheme versus the light-wave intensity. As for Fig. 2, $k v_{0}=50 \gamma$. The solid line corresponds to the numerical calculation based on accurate expressions (3) and (8). The dash-dotted blue line corresponds to the simplest approximate expression (18). The dashed red curve is obtained through the formula (16).

$$
A \approx \frac{2 \sqrt{\pi}}{x_{0}} I
$$

where the standard assumption $x_{0} \gg 1$ is taken into account. For the moderate intensities regime $(12 I \sim 1)$, we should slightly correct Eq. (17), coming to the formula :

$$
A \approx \frac{2 \sqrt{\pi}}{x_{0}} \frac{I}{\sqrt{1+12 I}}
$$

In the last case of relatively high intensities (from $I \approx 1$ up to several tens), we obtain a clear square-root dependence :

$$
A \approx \frac{\sqrt{\pi I / 3}}{x_{0}}
$$

The approximate solutions (16) and (18) are compared with the exact numerical calculation on Fig. 3. It is shown that both approximate analytical formula (16) and (18) lead to acceptable results for very small $\left(I \sim 10^{-4}\right.$ and lower $)$ and relatively high $(I \sim 10)$ intensities.

\section{B. Closed double $\Lambda$-scheme}

The closed double $\Lambda$-scheme can be thought analog to the closed single $\Lambda$-scheme in the case where the coherence $\rho_{34}$ (and $\rho_{43}=\rho_{34^{\star}}$ ) between the 3 and 4 upper-state levels, not induced in the single $\Lambda$-scheme (see Fig. 1a), is neglected (see Fig. 1b). Taking this coherence into account, we come for the closed double- $\Lambda$-scheme to the formula :

$$
W_{\Lambda-\Lambda}\left(x, \Delta_{R}\right)=\frac{16 I_{1} I_{2} \Delta_{R}^{2}}{b_{2} x^{2}+b_{1} x+b_{0}}
$$

with the coefficients :

$$
\begin{gathered}
b_{0}=16 I_{t}^{3}-8 \Delta_{R}^{2} I_{t}^{2}+\Delta_{R}^{2}\left(1+\Delta_{R}^{2}\right) I_{t}+48 \Delta_{R}^{2} I_{1} I_{2} \\
b_{1}=4 \Delta_{R}\left(I_{1}-I_{2}\right)\left[\Delta_{R}^{2}-4 I_{t}\right] \\
b_{2}=4 \Delta_{R}^{2} I_{t}
\end{gathered}
$$

We consider here $R_{1}$ to be the Rabi frequency for both $|1\rangle \rightarrow|3\rangle$ and $|1\rangle \rightarrow|4\rangle$ transitions, while $R_{2}$ is for $|2\rangle \rightarrow|3\rangle$ and $|2\rangle \rightarrow|4\rangle$ transitions. The same notations are used for $I_{t}$ and $I_{1,2}$. In this case, we obtain a result qualitatively similar to the one obtained for the single $\Lambda$-scheme, see Eqs. (3)-(6). It is worth noting that we can reduce the expressions (20)-(23) to the same expression than (3)-(6) just by replacing the intensities $I_{1,2}(\Lambda-\Lambda)=I_{1,2}(\Lambda) / 2$. Indeed, if we put $I_{1}(\Lambda-\Lambda)=I_{1}(\Lambda) / 2$ and $I_{2}(\Lambda-\Lambda)=I_{2}(\Lambda) / 2$ into Eqs. (20)-(23), we will come to $W_{\Lambda-\Lambda}=W_{\Lambda}$. While this operation might just seem an interesting mathematical trick, it has a real physical meaning. Indeed, by comparing the $\sigma^{+} \sigma^{+}$ light field configuration with the lin $\perp$ lin configuration [49], we can see that Rabi frequencies in these schemes are related as $R_{1,2}(\Lambda-\Lambda)=R_{1,2}(\Lambda) / 2^{1 / 2}$ due to cyclic components of the light polarization vectors. Therefore, the two polarization configurations $\sigma^{+} \sigma^{+}$and lin $\perp$ lin are identical for a vacuum vapour cell and closed system of levels.

Both previous subsections II-A and II-B have considered closed systems of atomic energy levels. This problem has a general academic interest and can be applied in specific experimental cases. However, in most experiments, the real atomic transitions induced by the light waves form an open system of levels (i.e. $\beta_{1}+\beta_{2}<1$ and, moreover, with $\beta_{1} \neq \beta_{2}$ ). Thus, the following subsection is dedicated to the study of a single open $\Lambda$-scheme.

\section{Single open $\Lambda$-scheme}

Many systems of atomic energy levels relevant for real experiments are open. In this configuration, one or more sub-levels are non-resonant with the light field. These sublevels can collect the atomic population during a long time and influence the properties of the nonlinear resonances. In 
particular, it is well-known that the openness can harm the amplitude of the dark resonance and impacts also the CPT resonance linewidth [60], [30].

Considering an open system of levels makes the problem much more complex in comparison with the closed one. An open system of levels is usually studied using the low light intensities regime, assuming that the "time-of-flight" saturation parameter, given in (9), is small enough. Here, we focus on a regime beyond this limit.

In this paper, we demonstrate the results for the resonance amplitude only. For shortness, we consider intensities to be equal $\left(I_{1}=I_{2}=I\right)$ as well as the branching ratios $\left(\beta_{1}=\beta_{2}=\beta\right)$. We also assume that $\beta_{1}+\beta_{2}<1$ and $\Gamma \neq 0$. We take $\Gamma \ll \gamma$ as a typical condition for a real experiment with thermal vapor. The branching ratios $\beta_{1,2}$ are assumed to be small $(\beta \ll 1)$. Eventually, one more important assumption is that $R^{2} / \gamma>\Gamma$, i.e. the power broadening prevails over the timeof-flight broadening. Using the same approach than in the previous paragraphs, we come to the following expression :

$$
\tilde{W}_{\Lambda}\left(x, \Delta_{R}\right)=\frac{p_{2} x^{2}+p_{0}}{q_{4} x^{4}+q_{2} x^{2}+q_{0}}
$$

with explicit expressions for the coefficients $p_{k}$ and $q_{k}$ reported in Appendix.

In this regime, due to the decoherence effect in the ground state $(\Gamma \neq 0)$, the fluorescent signal described in Eq. (24) is not equal to zero at $\Delta_{R}=0$, in contrast with (3) and (20). The amplitude can be found as follows :

$$
\tilde{A}=\tilde{W}_{\max }-\tilde{W}_{0}
$$

where $\tilde{W}_{\max }=\left\langle\tilde{W}_{\Lambda}\right\rangle_{v}\left(\Delta_{R}=\Delta_{R}^{\prime}\right)$ with $\Delta_{R}^{\prime}$ the position of the fluorescence maximum. Here, the latter does not equal to $\pm 2 R$ as for the closed scheme. $\tilde{W}_{0}=\left\langle\tilde{W}_{\Lambda}\right\rangle_{v}\left(\Delta_{R}=0\right)$ is the level of the signal at the center of the two-photon resonance.

Actually, the CPT effect can be "switched off" to calculate the first term in Eq. (25). This trick gives a quite acceptable result and means that the two light waves can be assumed to be mutually incoherent (for example, there could be two different laser sources without any phase lock). In this situation the low-frequency coherence between the ground states $|1\rangle$ and $|2\rangle$ is not induced (see Fig.1) and the dark resonance does not occur. To consider this regime in our theoretical model, we can just assume the low-frequency coherence (i.e. corresponding density matrix elements) to be null. Despite the use of this approximation, the analytical integration of Eq. (25) over the Maxwellian distribution still leads to a complex expression. Taking into account the assumptions mentioned in the beginning of this paragraph, the latter is simplified to :

$$
\tilde{W}_{\max } \approx \frac{\tilde{\Gamma} \xi}{(1-\beta)\left[x_{0}+(1-\beta) \xi\right]}
$$

where $\tilde{\Gamma}=\Gamma / \gamma$. A new time-of-flight saturation parameter (instead of the one given in Eq. (9)) has been introduced such that :

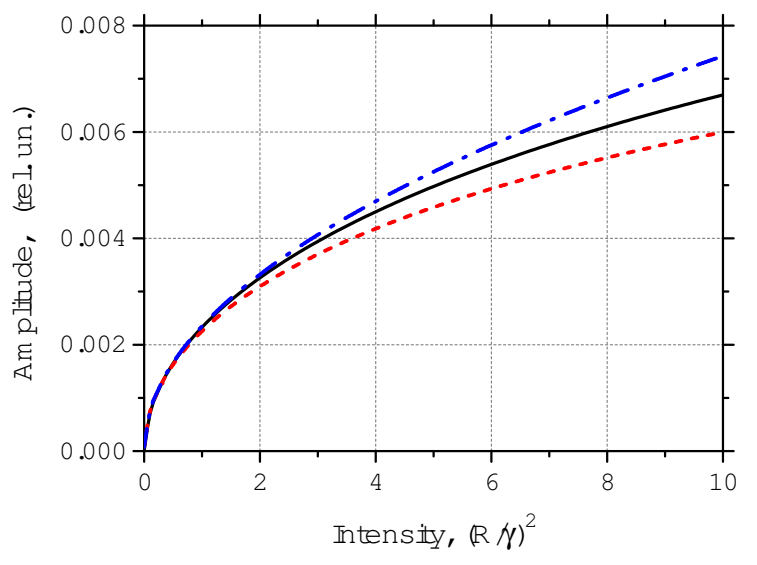

Fig. 4. Amplitude of the dark resonance in a vacuum vapour cell for a single open $\Lambda$-scheme versus the light-wave intensity. The Doppler halfwidth is $k v_{0}=50 \gamma$ and the time-of-flight relaxation rate is $\Gamma=0.02 \gamma$. The solid line corresponds to the numerical calculation on the basis of the expressions (8) and (24). The dash-dotted blue line corresponds to the simplest approximate expression (30). The red dashed curve represents the law (29).

$$
\xi=\sqrt{\pi \xi_{0}}=\sqrt{\pi \gamma \tau I}=\sqrt{\pi I / \tilde{\Gamma}}
$$

with $\xi_{0}$ used in Eq. (9).

For the center of the resonance, we obtain a very similar expression :

$$
\tilde{W}_{0} \approx \frac{\tilde{\Gamma} \xi}{\sqrt{2(1-\beta)}\left[x_{0}+\sqrt{2(1-\beta)} \xi\right]}
$$

Both expressions (26) and (28) give the amplitude :

$$
\begin{aligned}
\tilde{A} & \approx \frac{\tilde{\Gamma} \xi}{(1-\beta)}\left[\frac{1}{\left[x_{0}+(1-\beta) \xi\right]}-\right. \\
& \left.-\frac{\sqrt{1-\beta}}{\sqrt{2}\left[x_{0}+\sqrt{2(1-\beta)} \xi\right]}\right]
\end{aligned}
$$

Actually, under the low and moderate intensities (up to several tens of $\mathrm{mW} / \mathrm{cm}^{2}$ ), even if the condition $\xi \ll x_{0}$ is not satisfied, we can use a much simpler expression :

$$
\tilde{A} \approx\left(1-\sqrt{\frac{1-\beta}{2}}\right) \frac{\tilde{\Gamma} \xi}{x_{0}(1-\beta)}
$$

Let us now clarify the value of approximations (29) and (30) with the help of plots. In typical experiments using $\mathrm{Rb}$ or Cs thermal vapors and light beam diameters in the $1-10$ $\mathrm{mm}$ range, we approximately have $\Gamma \simeq(0.5-5) \times 10^{-2} \gamma$ and $x_{0}=k v_{0} / \gamma \approx 50$. The branching ratio, as explained further, is assumed to be $\beta=5 / 24$.

Figure 4 presents the approximate analytical expressions (29) and (30), together with the exact numerical calculation based on equations (24) and (8). Compared to the case of the closed $\Lambda$-scheme (Fig. 3), Fig. 4 reveals in the case of the open $\Lambda$ scheme a dramatic reduction of the CPT signal amplitude. As shown on Fig. 4, the obtained formula give quite good analytical approximations for the dark resonance amplitude in a wide range of light intensities $I=(R / \gamma)^{2}$. The simplest 
analytical expression, given in Eq. (30), can be used to estimate the CPT amplitude up to relatively large intensity values such that $I \approx 10$. Such values, corresponding to several hundreds of $\mathrm{mW} / \mathrm{cm}^{2}$, are not often used in the experiments.

\section{COMPARISON BETWEEN THEORY AND EXPERIMENTAL TESTS}

\section{A. Connexion of theory with experiments}

The present section III is devoted to comparison between theoretical and experimental results. In a first step, theoretical parameters like $I_{j}=\left(R_{j} / \gamma\right)^{2}, \Gamma$ etc. must be connected with those used in the experiments. In particular, during the derivation of expressions (29) and (30), we have assumed both ground-state sub-levels in the $\Lambda$-scheme to have initial populations equal to $1 / 2$. In the case of a real alkali atom, one should multiply (29) and (30) by the coefficient $\alpha$ defined as :

$$
\alpha=\left(F_{g 1}+F_{g 2}+1\right)^{-1}
$$

with $F_{g 1, g 2}$ the total angular momenta of the hyperfine levels in the ground state. For ${ }^{133} \mathrm{Cs}$, we have $\alpha=1 / 8$.

In present experiments, the CPT resonance is detected by monitoring the light power transmitted through the cell. With the help of Maxwell equations, the transmitted light wave intensity can be written in the form of quadratures :

$$
J_{L}\left(\Delta_{R}\right)=J_{0} \exp \left[-\int_{0}^{L} \eta(J(z)) d z\right]
$$

where $J_{0}$ is the incident light beam intensity and $J_{L}$ is the output intensity at the output of the cell, with $L$ the cell length. The function $\eta(z)$ is the absorption index per unit length. As mentioned in the introduction, the medium is considered to be optically thin. In such a case, the absorption index is small enough. Moreover, the latter does not depend significantly on the $\mathrm{z}$-coordinate. For this purpose, this dependence is neglected here. Therefore, we can write :

$$
J_{L}\left(\Delta_{R}\right) \approx J_{0}(1-\eta L)=J_{0}(1-\sigma n L),
$$

with $\sigma$ the absorption cross section and $n$ the atomic density in the cell. The optical Bloch equations lead to :

$$
\sigma=\frac{2 \pi c \hbar \gamma}{\lambda} \times \frac{\left\langle\tilde{W}_{\Lambda}\right\rangle_{v}\left(J_{0}\right)}{J_{0}}
$$

with $c$ the speed of light and $\hbar=h /(2 \pi)$ with $h$ the Planck constant and $\tilde{W}_{\Lambda}$ extracted from (24).

The dark resonance amplitude $\tilde{A}_{T}$ observed in the transmitted signal $J_{L}\left(\Delta_{R}\right)$ is determined by the expression :

$$
\tilde{A}_{T}=J_{0} n L\left(\sigma_{\max }-\sigma_{0}\right) .
$$

Here $\sigma_{0}$ is the cross section at the center of resonance $\left(\Delta_{R}=\right.$ $0), \sigma_{\max }$ corresponds to the maximum level of the light wave absorption and defines a wide background pedestal of the dark resonance.

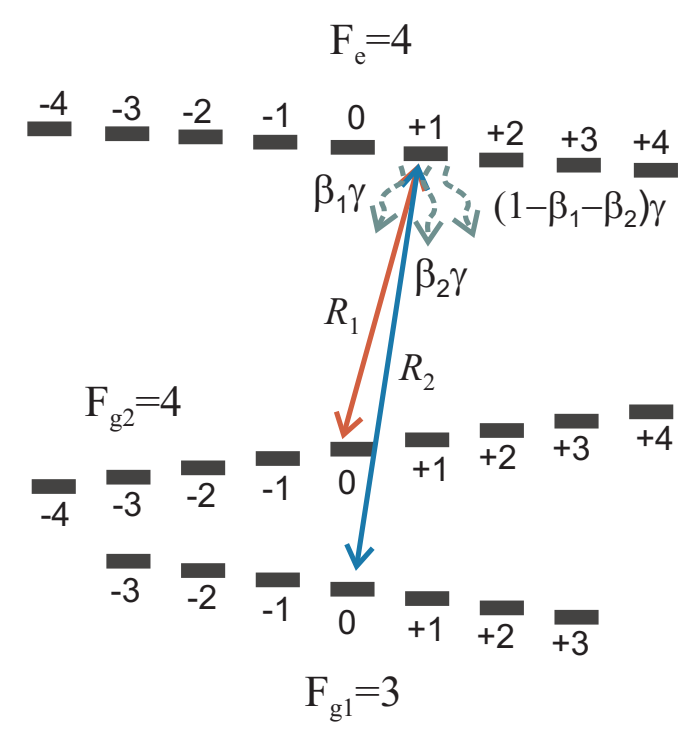

Fig. 5. Partial structure of energy levels involved in the Cs $D_{1}$ line (not in scale). The magnetic field is switched on. Numbers correspond to the quantum numbers $m$. Straight solid arrows denote $\sigma^{+}$light induced transitions. Wavy dashed arrows show three possible channels for the spontaneous decay with corresponding branching ratios $\beta . R_{1,2}$ are the Rabi frequencies for both arms of the open single $\Lambda$-scheme.

Involving the approximate solution (30), expressions (34) and (35) together with (25) and (31) result in the expression :

$$
\tilde{A}_{T}=\frac{2 \pi \alpha c \hbar \gamma n L}{\lambda x_{0}(1-\beta)} \times\left(1-\sqrt{\frac{1-\beta}{2}}\right) \sqrt{\pi \tilde{\Gamma} I} .
$$

The experimental intensity $J$ is defined from the intensity $I$ used in the theory such that :

$$
I \equiv\left(\frac{R}{\gamma}\right)^{2}=\frac{J}{8 J_{s}} \times \Psi,
$$

where $J_{S}$ is the saturation intensity determined here in the following way [61] :

$$
J_{S}=\frac{2 \pi^{2} \hbar c \gamma}{3 \lambda^{3}}
$$

For cesium $\mathrm{D}_{1}$ line, we get $J_{s} \approx 7900 \mathrm{erg} / \mathrm{s} / \mathrm{cm}^{2}=0.79$ $\mathrm{mW} / \mathrm{cm}^{2}$. The parameter $\Psi$ in (37) contains information about the relative strength of a certain dipole transition $\left|F_{g}, m_{g}\right\rangle \rightarrow$ $\left|F_{e}, m_{e}\right\rangle$ with $m_{g, e}$ the magnetic quantum numbers of the ground $(g)$ and excited $(e)$ states (see Fig.5):

$$
\begin{aligned}
\Psi & =\left(2 J_{e}+1\right)\left(2 F_{g}+1\right)\left(2 F_{e}+1\right) \times \\
& \times\left(\begin{array}{ccc}
F_{e} & 1 & F_{g} \\
-m_{e} & 1 & m_{g}
\end{array}\right)^{2}\left\{\begin{array}{lll}
J_{e} & F_{e} & I_{n} \\
F_{g} & J_{g} & 1
\end{array}\right\}^{2} .
\end{aligned}
$$

Here $I_{n}$ is the nucleus spin. $J_{g}, J_{e}$ are the total angular momenta of electrons for the ground and excited states respectively. Since the $\sigma^{+} \sigma^{+}$excitation configuration is studied, we took $q=1$ in Eq. (39), with $q$ the light polarization component in the circular basis (in general, $q= \pm 1,0$ ). The standard notations $(\ldots)$ and $\{\ldots\}$ for $3 j m-$ and $6 j-$ symbols are used [62].

Figure 5 shows the partial structure of energy levels of the 
Cs $\mathrm{D}_{1}$ line $\left(F_{e}=4\right.$ excited state). The strength $\Psi=5 / 24$ is the same for both transitions induced in the $\Lambda$-scheme shown in Fig. 5. Therefore, as already assumed above, $I_{1}=I_{2} \equiv I$. The total intensity of the light field is $I_{t}=2 I\left(J_{t}=2 J\right)$. Expression (39) coincides with the effective branching ratio for a single transition of the $\Lambda$-scheme. The branching ratios for both transitions of the scheme are equal to $\beta_{1}=\beta_{2}=5 / 24$. Expressions (36)-(38) lead to the square-root law dependence of the CPT amplitude to the intensity $J$, such as :

$$
\tilde{A}_{T}=\frac{3 \alpha \lambda^{2} n L}{4 x_{0}}\left(\sqrt{\frac{2}{1-\beta}}-1\right) \times \sqrt{\frac{\tilde{\Gamma} J_{S} \Psi}{2 \pi(1-\beta)} J_{t}}
$$

\section{B. Description of the experimental setup}

We have implemented an experimental setup, similar to the one described in [63], to perform CPT spectroscopy in a Cs vapor microfabricated cell.

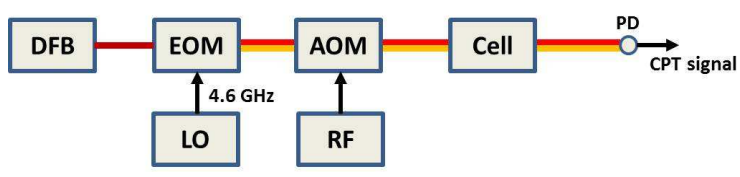

Fig. 6. Experimental setup to perform CPT spectroscopy. DFB : distributed feedback laser, EOM : Mach-Zehnder electro-optic modulator, LO : local oscillator, AOM : acousto-optic modulator, RF : radiofrequency synthesizer, Cell : cell under test, PD : photodiode

The laser source is a $1 \mathrm{MHz}-$ linewidth distributed feedback (DFB) diode laser, tuned on the $\mathrm{Cs}_{1}$ line at $895 \mathrm{~nm}$. A $70 \mathrm{~dB}$ optical isolation stage is implemented at the output of the laser source to prevent optical feedback. The laser light is injected into a fibered Mach-Zehnder EOM (EOM iXblue Photline NIR-MX800-LN-10). The EOM is driven by a microwave frequency synthesizer at $4.596 \mathrm{GHz}$ such that both first-order optical sidebands are frequency-split by $9.192 \mathrm{GHz}$ and can be used for CPT interaction. Dedicated servo systems are used to reduce greatly the optical power contained in the optical carrier and higher-order harmonics. This allows to obtain a clean optical spectrum, composed roughly of only two optical lines. At the output of the EOM, the light is sent into an acousto-optical modulator which can be used to control the laser power. The light is then sent into a quarter-wave plate to obtain a circularly-polarized laser beam and crosses a 2$\mathrm{mm}$ diameter and 1.4-mm length $\mathrm{Cs}$ vapor microfabricated cell [64]. The beam diameter has been measured to be 1.21 $\mathrm{mm}$ using a beam profiler. The cell is temperature-stabilized at $75^{\circ} \mathrm{C}$. On the basis of measured linear absorption profiles, the atomic density was estimated to be about $1.2 \times 10^{12} \mathrm{at}^{\mathrm{cm}} \mathrm{cm}^{-3}$. At this temperature, the optical absorption is measured to be about $40 \%$, corresponding to a non-negligible optical thickness. A static magnetic field of $10.4 \mathrm{G}$ is applied in order to separate well Zeeman transitions and isolate the 00 clock two-photon transition. During the experiments, the laser frequency is connected to the $|F=4\rangle$ excited state. The frequency of the microwave frequency synthesizer is swept over a few $\mathrm{MHz}$ in order to detect the CPT resonance in the bottom of the CPT-resonant absorption line. Once recorded, the CPT resonance is fitted by a Lorentzian function in order to extract its main characteristics (linewidth and amplitude). Such measurements are performed for a wide range of laser intensities, up to about $60 \mathrm{~mW} / \mathrm{cm}^{2}$.

\section{Experimental results and comparison with theory}

Figure 7 plots the evolution of the CPT amplitude versus the laser intensity, in the case of a circular polarization scheme.

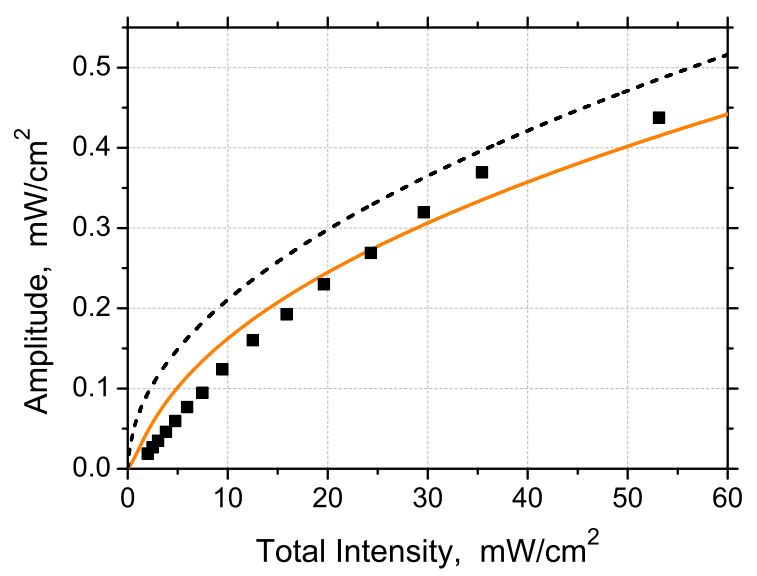

Fig. 7. Comparison between theoretical curves and experimental data (black squares). Amplitude of the CPT resonance versus the total laser intensity $J_{t}$ of the laser field. The solid orange line is for numerical calculations on the basis of equations (24) and (8). The time-of-flight relaxation constant is taken to be $\Gamma=0.06 \gamma$. The dashed black line shows the result of the analytical expression reported in (40).

Experimental results are compared with theoretical expectations extracted from analytical results and numerical simulations. Numerical calculations are found to be in correct agreement with the experimental data. The agreement between analytical formula and experimental data is less satisfying. Several reasons can explain this discrepancy. The first suggestion is that the optical thickness of the medium is not negligible in our experimental test. The medium can not be fully considered as an optically thin medium as assumed in the theoretical section. The second and the most valuable reason is that the calculation a priori of an appropriate value for the time-of-flight relaxation constant $\Gamma$ is difficult. Indeed, our theory relies on the standard theoretical approach of relaxation constants, often used to obtain analytical results [28], [30], [29]. However, strictly speaking, the validity of this approach is higher for light beams with a large-enough beam diameter and exhibiting a step-like intensity profile. In the latter case, the constant $\Gamma$ can be estimated on the basis of well-known expressions (for instance, see [61]). The acceptable results can be also obtained for the case of small time-of-flight saturation parameter (9) [59]. At the same time, it is well known that in the general case the relaxation-constants approach may demonstrate a significant discrepancy with either real experimental observations or accurate numerical calculations taking into account the transit-time effect under the real transverse laser intensity distribution (e.g., see [45], [65], [66]). This problem can be really relevant in the case where a narrow Gaussian beam is used, as actually operated in our experimental setup. Additionally, our situation is even more complicated through 
the use of a miniature vapor cell, which does not allow us using larger beam diameters (5-10 $\mathrm{mm}$ instead of $1.2 \mathrm{~mm})$. Under these conditions, the assumption in the theoretical section that the power broadening is the dominant contribution to the CPT line shape might be distorted due to the interaction with a beam of finite width [43]. In particular, this is why the relative discrepancy between the dashed curve and black squares in Fig. 7 is more noticeable at low light intensity levels than at the high ones. Nevertheless, we consider that the agreement between theory and experiments is correct, so that the derived analytical expression (40) can be used for estimating the amplitude of CPT resonance in practice.

A last possible reason to explain the discrepancy between experimental data and the model comes from our theoretical model in which the multi-level Cs $\mathrm{D}_{1}$ structure is simplified to a 3-level $\Lambda$-model. For example, the particular groundstate sub-level $\left|F_{g}=4, m=0\right\rangle$ in Fig. 5 experiences additional optical pumping due to spontaneous emission from excitedstate sub-levels $\left|F_{e}=4, m=0\right\rangle$ and $\left|F_{e}=4, m=-1\right\rangle$. Such processes are not considered in the $\Lambda$-scheme approach used in our theory. Nevertheless, we consider that experimental and theoretical results fit correctly and that the derived analytical expression Eq. (40) can be used for estimating the amplitude of CPT resonance.

To summarize aforesaid, we can state that in our configuration the relaxation rate $\Gamma$ can be considered as a matching parameter to get a good agreement between theory and experiments. In particular, the numerical calculation presented in Fig. 7 has been performed with $\Gamma=0.06 \gamma$. This value gives a good agreement between the numerical calculation and experimental data but this regime remains pretty far from the one required in the theoretical section. We anticipate that a better agreement between theory and experiments should be obtained using a laser beam with a larger diameter and a cm-size vapor cell. In this case, the $\Gamma$ rate could be estimated a priori as described in [61].

\section{CONCLUSIONS}

We have reported a theoretical and experimental study on the properties (amplitude and linewidth) of CPT resonances detected in a vacuum vapor cell, using single or double $\Lambda$ schemes. Theoretical results have been obtained in the case the closed single and double $\Lambda$-schemes. In the case of an open single $\Lambda$-scheme, the CPT amplitude vs. light intensity dependence has been derived and presented in a clear compact form for the first time. A main feature of the reported approximate analytical expressions is their validity for a wide range of light wave intensities. Analytical expressions demonstrate a good agreement with exact numerical solutions until the power broadening prevails over the other broadening mechanisms and until the atomic vapor can be assumed as an optically thin medium. Experimental results (CPT amplitude), obtained by performing CPT spectroscopy in an evacuated Cs vapor microfabricated cell have been reported. A good agreement with exact numerical calculations is reported. Comparisons between experimental data and analytical expressions for the CPT amplitude are found to be acceptable. A better agreement should be obtained using a regular cm-size vapor cell and a larger beam diameter.

\section{ACKNOWLEDGMENTS}

The authors thank Dr. E. De Clercq (LNE-SYRTE, Paris) and Prof. A. Taichenachev (ILP SB RAS, Novosibirsk) for stimulating discussions.

\section{APPENDIX}

The explicit form for the coefficients in (24) is :

$$
\begin{aligned}
& p_{0}=4 R^{2} \Gamma\left[4 R^{2} \Gamma+\gamma\left(\Gamma^{2}+\Delta_{R}^{2}\right)\right] \times \\
& \times\left[4 R^{2} \gamma+\Gamma\left(\gamma^{2}+\Delta_{R}^{2}\right)\right], \\
& p_{2}=16 R^{2} \Gamma^{2} \gamma^{3}\left(\Gamma^{2}+\Delta_{R}^{2}\right), \\
& q_{0}=\left[4 R^{2} \gamma+\Gamma\left(\gamma^{2}+\Delta^{2}\right)\right]\left\{32 R^{4} \Gamma(2 \Gamma+\gamma(1-\beta))+\right. \\
& +\Gamma(\gamma+\Gamma)\left(\gamma^{2}+\Delta_{R}^{2}\right)\left(\Gamma^{2}+\Delta_{R}^{2}\right)+ \\
& +4 R^{2}\left[\gamma \Gamma^{2}(5 \Gamma+\gamma(3-2 \beta))+\right. \\
& \left.\left.+\Delta^{2}\left(\gamma^{2}(1-2 \beta)+\Gamma(\gamma-2 \Gamma)\right)\right]\right\}, \\
& q_{2}=8 \Gamma \gamma^{2}\left\{\Gamma(\gamma+\Gamma)\left[8 R^{4}+\left(\gamma^{2}-\Delta_{R}^{2}\right)\left(\Gamma^{2}+\Delta_{R}^{2}\right)\right]+\right. \\
& \left.+4 R^{2}\left[\Gamma^{2} \gamma(3 \Gamma+\gamma(2-\beta))+\Delta_{R}^{2}\left(\gamma^{2}(1-\beta)+\Gamma(3 \gamma+\Gamma)\right)\right]\right\}, \\
& q_{4}=16 \Gamma^{2} \gamma^{4}(\gamma+\Gamma)\left(\Delta_{R}^{2}+\Gamma^{2}\right) \text {. }
\end{aligned}
$$

\section{REFERENCES}

[1] G. Alzetta, "An experimental method for the observation of RF transitions and laser beat resonances in oriented Na vapor" Nuovo Cimento B, vol. 36, pp. 5-20 1976.

[2] E. Arimondo and G. Orriols, "Non-absorbing atomic coherences by coherent two-photon transitions in a three-level optical pumping," Lett. Nuovo Cimento, vol. 17, pp. 333-338, 1976.

[3] R. G. Brewer and E. L. Hahn, "Coherent two-photon processes : Transit and steady-state cases," Phys. Rev. A, vol. 11, pp. 1641-1649, 1975.

[4] H. R. Gray, R. M. Whitley and C. R. Stroud Jr., "Coherent trapping of atomic populations," Opt. Lett., vol. 3, pp. 218-220, 1978.

[5] E. Arimondo, "Coherent population trapping in laser spectroscopy," Progress in Optics, vol. 35, pp. 257-354, 1996.

[6] R. Wynands and A. Nagel, "Precision spectroscopy with coherent dark states," Appl. Phys. B, vol. 68, pp. 1-25, 1999.

[7] J. Vanier, "Atomic clocks based on coherent population trapping : a review," Appl. Phys. B, vol. 81, pp. 421-442, 2005.

[8] A. Nagel, L. Graf, A. Naumov, E. Mariotti, V. Biancalana, D. Meschede, and R. Wynands, "Experimental realization of coherent dark-state magnetometers," Europhys. Lett., vol. 44, no. 1, pp. 31-36, 1998.

[9] P. D. D. Schwindt, S. Knappe, V. Shah, L. Hollberg and J. Kitching, "Chip-scale atomic magnetometer," Appl. Phys. Lett., vol. 85, no. 26, pp. 6409-6411, 2004.

[10] A. Aspect, E. Arimondo, R. Kaizer, N. Vansteenkiste and C. CohenTannoudji, "Laser cooling below the one-photon recoil energy by velocityselective coherent population trapping : theoretical analysis," J. Opt. Soc. Am. B, vol. 6, no. 11, pp. 2112-2124, 1989. 
[11] M. Bajcsy, A. S. Zibrov and M. D. Lukin, "Stationary pulses of light in an atomic medium," Nature, vol. 426, pp. 638-641, 2003.

[12] M. Bayer, "Coherent population trapping : Quantum optics with dots," Nature Physics, vol. 4, pp. 678-679, 2008.

[13] J. Vanier and C. Audoin, "The quantum physics of atomic frequency standards," Adam Hilger, Bristol, 1989.

[14] R. H. Dicke, "The Effect of Collisions upon the Doppler Width of Spectral Lines," Phys. Rev., vol. 89, no. 2, pp. 472-473, 1953.

[15] E. Bernabeu and J. M. Alvarez, "Shift and broadening of hyperfine components of the first doublet of cesium perturbed by foreign gases," Phys. Rev. A, vol. 22, no. 6, pp. 2690-2695 (1980).

[16] A. H. Couture, T. B. Clegg and B. Driehuys, "Pressure shift and broadening of the $C s \mathrm{D}_{1}$ and $\mathrm{D}_{2}$ lines by $\mathrm{He}, \mathrm{N}_{2}$ and $\mathrm{Xe}$ at densities used for optical pumping and spin exchange polarization," J. Appl. Phys., vol. 104, 094912, 2008

[17] G. A. Pitz, D. E. Wertepny and G. P. Perram, "Pressure broadening and shift of the Cs $\mathrm{D}_{1}$ transition by the noble gases and $\mathrm{N}_{2}, \mathrm{H}_{2}, \mathrm{HD}, \mathrm{D}_{2}$, $\mathrm{CH}_{4}, \mathrm{C}_{2} \mathrm{H}_{6}, \mathrm{CF}_{4}$ and ${ }^{3} \mathrm{He}, "$ Phys. Rev. A, vol. 80, 062718, 2009.

[18] J. Vanier, R. Kunski, N. Cyr, J. Y. Savard and M. Têtu, "On hyperfine frequency shifts caused by buffer gases : Application to the optically pumped passive rubidium frequency standard," J. Appl. Phys., vol. 53, no. 8, pp. 5387-5391, 1982.

[19] O. Kozlova, S. Guérandel and E. De Clercq, "Temperature and pressure shift of the Cs clock transition in the presence of buffer gases : $\mathrm{Ne}, \mathrm{N}_{2}$, Ar," Phys. Rev. A, vol. 83, 6062714, 2011.

[20] E. Kroemer, M. Abdel Hafiz, V. Maurice, B. Fouilland, C. Gorecki and R. Boudot, "Cs vapor microcells with Ne-He buffer gas mixture for high operation-temperature miniature atomic clocks" Opt. Exp., vol. 23, no. 14, pp. 18373-18380, 2015.

[21] D. Miletic, P. Dziuban, R. Boudot, M. Hasegawa, R. K. Chutani, G. Mileti, V. Giordano and C. Gorecki, "Quadratic dependence on temperature of Cs 0-0 hyperfine resonance frequency in single $\mathrm{Ne}$ buffer gas microfabricated vapour cell," Elec. Lett., vol. 46, no. 15, pp. 10691071, 2010.

[22] M. A. Bouchiat and J. Brossel, "Relaxation of optically pumped Rb atoms on paraffin-coated walls", Phys. Rev., vol. 147, no. 1, pp. 41-54, 1966.

[23] M. T. Graf, D. F. Kimball, S. M. Rochester, K. Kerner, C. Wong, D. Budker, E. B. Alexandrov, M. V. Balabas and V.V. Yashchuk, "Relaxation of atomic polarization in paraffin-coated cesium vapor cells," Phys. Rev. A, vol. 72, 023401, 2005.

[24] S. J. Seltzer, P. J. Meares and M. V. Romalis, "Synchronous optical pumping of quantum revival beats for atomic magnetometry," Phys. Rev. A, vol. 75, 051407(R), 2007.

[25] S. J. Seltzer, D. M. Rampulla, S. Rivillon-Army, Y. J. Chabal, S. L. Bernasek and M. V. Romalis, "Testing the effect of surface coatings on alkali atom polarization lifetimes," J. Appl. Phys., vol. 104, 103116, 2008.

[26] M. Abdel Hafiz, V. Maurice, R. Chutani, N. Passilly, C. Gorecki, S. Guérandel, E. de Clercq and R. Boudot, "Characterization of Cs vapor cell coated with octadecyltrichlorosilane using coherent population trapping spectroscopy", J. Appl. Phys., vol. 117, 184901, 2015.

[27] M. V. Balabas, T. Karaulanov, M. P. Ledbetter and D. Budker, "Polarized Alkali-Metal Vapor with Minute-Long Transverse Spin-Relaxation Time", Phys. Rev. Lett., vol. 105, 070801, 2010.

[28] A. V. Taichenachev, A. M. Tumaikin, and V. I. Yudin, "Influence of atomic motion on the shape of two-photon resonance in gas," JETP Lett., vol. 72, no. 3, pp. 119-122, 2000.

[29] C. Y. Ye and A. S. Zibrov, "Width of the electromagnetically induced transparency resonance in atomic vapor," Phys. Rev. A, vol. 65, 023806, 2002.

[30] A. Javan, O. Kocharovskaya, H. Lee, and M.O. Scully, "Narrowing of electromagnetically induced transparency resonance in a Dopplerbroadened medium," Phys. Rev. A, vol. 66, 013805, 2002

[31] H. Failache, L. Lenci, A. Lezama, D. Bloch, and M. Ducloy, "Theoretical study of dark resonances in micrometric thin cells," Phys. Rev. A, vol. 76, 053826, 2007

[32] B. J. Dalton and P. L. Knight, "The effects of laser field fluctuations on coherent population trapping," J. Phys. B : At. Mol. Opt. Phys., vol. 15, no. 21 , p. 3997,1982

[33] R. M. Guo, F. Xiao, C. Liu, Y. Zhang and X-Z. Chen, Chin. Phys. Lett. 20, 1507 (2003).
[34] E. Breschi, G. Kazakov, R. Lammegger, B. Matisov, L. Windholz and G. Mileti, "Influence of Laser Sources with Different Spectral Properties on the Performance of Vapor Cell Atomic Clocks Based on lin $\|$ lin CPT," IEEE Trans. Ultrason. Ferroelec. Freq. Contr., vol. 56, no. 5, pp. 926-930, 2009.

[35] G. Kazakov, B. Matisov, A. Litvinov and I. Mazets, "Coherent population trapping in a finite-size buffer-less cell," Journ. Phys. B : At. Mol. Opt. Phys., vol. 40, no. 19, p. 3851, 2007.

[36] S. Knappe, MEMS Atomic Clocks, Comprehensive Microsystems, Elsevier B.V, vol. 3, pp. 571-612, 2006.

[37] R. Lutwak, A. Rashed, M. Varghese, G. Tepolt, J. Leblanc, M. Mescher, D. K. Serkland and G. M. Peake, "The miniature atomic clock : preproduction results," in Proc. IEEE International Freq. Contr. Symposium Eur. Frequ. Time Forum. Joint Meeting, Geneva, Switzerland, pp. 13271333, 2007.

[38] M. Abdel Hafiz, G. Coget, E. de Clercq and R. Boudot, "Doppler-free spectroscopy on the $\mathrm{Cs}_{1} \mathrm{D}_{1}$ line with a dual-frequency laser," Opt. Lett., vol. 41, no. 13, pp. 2982-2985, 2016.

[39] M. Abdel Hafiz, D. Brazhnikv, G. Coget, A. Taichenachev, V. Yudin, E. de Clercq and R. Boudot, "High-contrast sub-Doppler absorption spikes in a hot atomic vapor cell exposed to a dual-frequency laser field," New J. Phys., vol. 19, 073028, 2017.

[40] P. L. Kelley, P. J. Harshman, O. Blum, and T. K. Gustafson, "Radiative renormalization analysis of optical double resonance," J. Opt. Soc. Am. $B$, vol. 11, no. 11, pp. 2298-2302, 1994.

[41] B. D. Agap'ev, M. B. Gornyi, B. G. Matisov and Yu. V. Rogdestvenskii, "Coherent population trapping in quantum systems," Physics-Uspekhi, vol. 36, pp. 763-793 (1993).

[42] T. Zanon-Willette, E. de Clercq and E. Arimondo, "Ultrahigh-resolution spectroscopy with atomic or molecular dark resonances : Exact steadystate line shapes and asymptotic profiles in the adiabatic pulsed regime," Phys. Rev. A, vol. 84, 062502, 2011.

[43] J. E. Thomas and W. W. Quivers, Jr., "Transit-time effects in optically pumped coupled three-level systems," Phys. Rev. A, vol. 22, no. 5, p. 2115, 1980.

[44] J. Vanier, A. Godone, and F. Levi, "Coherent population trapping in cesium : Dark lines and coherent microwave emission," Phys. Rev. A, vol. 58, no. 3, pp. 2345-2358, 1998.

[45] A. V. Taichenachev, A. M. Tumaikin, V. I. Yudin, M. Stähler, R. Wynands, J. Kitching, and L. Hollberg, "Nonlinear-resonance line shapes Dependence on the transverse intensity distribution of a light beam," Phys. Rev. A, vol. 69, 024501, 2004.

[46] A. V. Taichenachev, V. I. Yudin, R. Wynands, M. Stähler, J. Kitching, and L. Hollberg, "Theory of dark resonances for alkali-metal vapors in a buffer-gas cell," Phys. Rev. A, vol. 67, 033810, 2003.

[47] S. Khan, M. P. Kumar, V. Bharti, and V. Natarajan, "Coherent population trapping (CPT) versus electromagnetically induced transparency (EIT)," Eur. Phys. J. D., vol. 71, no. 38, 2017.

[48] R. Malitskiy, V. M. Khodakovskiy, A. M. Negriyko, V. I. Romanenko, I. V. Matsnev, and L. P. Yatsenko, "Magneto-optical CPT resonances in rubidium excited by femtosecond laser comb," Proc. of SPIE, International Conference on coherent and nonlinear optics (ICONO 2010) and international conference on lasers, applications and technologies (LAT 2010), 23-27 August 2010, Kazan, Russian Federation 7993, 799312 2011.

[49] T. Zanon, S. Guérandel, E. de Clercq, D. Holleville, N. Dimarcq and A. Clairon, "High contrast Ramsey fringes with coherent-population-trapping pulses in a double lambda atomic system," Phys. Rev. Lett., vol. 94, 193002, 2005

[50] S. A. Zibrov, V. L. Velichansky, A. S. Zibrov, A. V. Taichenachev and V. I. Yudin, "Experimental investigation of the dark pseudoresonance on the $\mathrm{D}_{1}$ line of the ${ }^{87} \mathrm{Rb}$ atom excited by a linearly polarized field," JETP Lett., vol. 82, no. 8, pp. 477-481 (2005).

[51] Y. Y. Jau, E. Miron, A. B. Post, N. N. Kuzma and W. Happer, "Push-pull optical pumping of pure superposition states," Phys. Rev. Lett., vol. 93, 160802, 2004

[52] X. Liu, J. M. Mérolla, S. Guérandel, C. Gorecki, E. de Clercq and R. Boudot, "Coherent-population-trapping resonances in buffer-gas-filled Cs-vapor cells with push-pull optical pumping," Phys. Rev. A, vol. 87, 013416, 2013.

[53] P. Yun, J. M. Danet, D. Holleville, E. de Clercq and S. Guérandel, "Constructive polarization modulation for coherent population trapping clock," Appl. Phys. Lett., vol. 105, 231106, 2014. 
[54] M. Abdel Hafiz, G. Coget, P. Yun, S. Guérandel, E. de Clercq an R. Boudot, "A high-performance Raman-Ramsey Cs vapor cell atomic clock," J. Appl. Phys. vol. 121, 104903, 2017.

[55] P. Yun, F. Tricot, C. E. Calosso, S. Micalizio, B. Francois, R. Boudot, S. Guérandel and E. de Clercq, "High-Performance Coherent Population Trapping Clock with Polarization Modulation," Phys. Rev. Applied, vol. 7, $014018,2017$.

[56] E. Breschi, S. D. Gruji, P. Knowles and A. Weis, "A high-sensitivity push-pull atomic magnetometer," Appl. Phys. Lett., vol. 104, 023501, 2014.

[57] S. G. Rautian and A. M. Shalagin, "Kinetic Problems of Nonlinear Spectroscopy," Amsterdam : Elsevier, 1991.

[58] A. Godone, F. Levi, S. Micalizio, and J. Vanier, "Dark-line in opticallythick vapors : inversion phenomena and line width narrowing," Eur. Phys. J. $D$, vol. 18 , pp. $5-13,2002$

[59] D. B. Lazebnyi, D.V. Brazhnikov, A.V. Taichenachev, M.Yu. Basalaev, and V.I. Yudin, "Electromagnetically induced absorption and electromagnetically induced transparency for optical transitions $F_{g} \rightarrow F_{e}$ in the field of elliptically polarized waves," J. Exp. Theor. Phys., vol. 121, pp. 934-949, 2015.

[60] F. Renzoni, A. Lindner, and E. Arimondo, "Coherent population trapping in open systems : A coupled/noncoupled-state analysis," Phys. Rev. A, vol. 60, no. 1, pp. 450-455, 1999.

[61] F. Riehle, "Frequency Standards : Basics and Applications." Weinheim : Wiley-VCH, p. 149, 2004.

[62] D.A. Varshalovich, A.N. Moskalev and V.K. Khersonskii, "Quantum Theory of Angular Momentum". Singapore : World Scientific, 1989.

[63] M. Abdel Hafiz and R. Boudot, "A coherent population trapping Cs vapor cell atomic clock based on push-pull optical pumping," J. Appl. Phys., vol. 118, 124903, 2015.

[64] M. Hasegawa, R. K. Chutani, C. Gorecki, R. Boudot, P. Dziuban, V. Giordano, S. Clatot and L. Mauri, "Microfabrication of cesium vapor cells with buffer gas for MEMS atomic clocks," Sensors and Actuators A, vol. 167, pp. 594-601, 2011.

[65] S.N. Bagayev, V.P. Chebotayev, and E.A. Titov, "Saturated absorption lineshapes under the transit-time conditions," Laser Physics, vol. 4, pp. 224-292, 1994.

[66] M. Radonjić, D. Arsenović, Z. Grujić, and B. M. Jelenković, "Coherent population trapping linewidths for open transitions : Cases of different transverse laser intensity distribution," Phys. Rev. A, vol. 79, 023805, 2009 .

Denis V. Brazhnikov was born in Novosibirsk, Russia, in 1982. He received the master of physics degree from Novosibirsk State Technical University in 2006, and the Ph.D. degree in physics from Institute of Laser Physics, Siberian Branch of Russian Academy of Sciences in 2009. He is currently an associate professor in the Physics Department of Novosibirsk State University and a senior researcher in the ILP SB RAS. His research interests include theoretical aspects of high-resolution laser spectroscopy of atoms, coherent population trapping and related effects, atomic clocks, magnetometers and laser cooling of neutral atoms.

Grégoire Coget was born in Caen, France, in 1991. He received the Master degree of Physics (option : Photonics) in 2015 from Université de Rennes, France. He's now pursuing a PhD degree at FEMTO-ST, Besançon, France, working on a high-performance pulsed Cs vapor cell CPT clock. His research interests include time-frequency metrology, CPT-based vapor cell atomic clocks and high-resolution laser spectroscopy.

Moustafa Abdel Hafiz was born in Paris, France, in 1989. He received the Master of physics (Option : quantum devices) from Université Paris 7, France in 2012. He received his Ph.D. degree in 2017 from Université de FrancheComté, Besançon, France. His thesis, performed at FEMTO-ST, Besançon, France, dealt with the development and metrological characterization of a high-performance CPT-based Cs cell clock.
Vincent Maurice was born in Langres, France, in 1988. He received his Ph.D. degree in 2016 from Université de Franche-Comté, Besançon, France. His thesis, performed at FEMTO-ST, Besançon, France, dealt with the design, microfabrication and characterization of alkali vapor cells for miniature atomic frequency references. V. Maurice is now working as a post-doctoral researcher at NIST, Boulder, in Atomic Devices and Instrumentation (ADI) Group.

Christophe Gorecki is a Director of Research (DR1) at CNRS. He received the Ph.D. degree in Optics from Université de Franche-Comté, Besançon, France in 1983. He joined the CNRS in 1984 as a CNRS Scientist. His research interests included optical inspection and micro-measurements, applications of image processing techniques in optical metrology and Optical Pattern Recognition methods. From 1995 to 1998, he worked at the University of Tokyo where he started to develop MOEMS architectures for microand nano-sensors. He has to his credit more than 200 technical papers and three book chapters. He has been a reviewer for several international journals and has organized and chaired various SPIE conferences. He is a Fellow of the SPIE and the elected member of SPIE Board of Directors. He has supervised several European and national projects in the field of micro and nanotechnologies and coordinated the collaborative project MACTFC, dedicated to the development of a European miniature atomic clock. C. Gorecki has received in 2012 the annual prize of the European Society of Optics.

Rodolphe Boudot was born in Dijon, France, in 1980. He received the M.S degree in electronics in 2003 and the Ph.D. degree in engineering sciences in 2006, both from Université de Franche-Comté, Besançon, France. His PhD thesis, performed at FEMTO-ST, Besançon, France, dealt with the development and metrology of low phase noise microwave sapphire oscillators. From 2007 to 2009, R. Boudot spent a 2-year post-doctoral position at the Systèmes de Référence Temps-Espace laboratory (SYRTE, Paris, France), working on a pulsed Cs vapor cell clock based on coherent population trapping (CPT). Since October 2008, R. Boudot has been a permanent CNRS researcher at FEMTO$\mathrm{ST}$. His research interests include CPT-based vapor cell atomic clocks, low noise electronics, oscillators and frequency synthesizers, high-resolution laser spectroscopy and more recently cold-atoms and ion-trapped atomic clocks. 